\title{
Influence of Electromagnetic Interactions on the Line Shape of Plasmonic Fano Resonances
}

\author{
Benjamin Gallinet and Olivier J. F. Martin* \\ Nanophotonics and Metrology Laboratory, Swiss Federal Institute of Technology (EPFL), CH-1015 Lausanne
}

$\mathrm{T}$ hanks to their ability to generate extremely strong and confined optical fields at the nanoscale, resonant metallic nanostructures supporting localized surface plasmon polariton modes play a remarkable role in current nanoscience. ${ }^{1} \mathrm{Re}$ cently, it has been shown that a proper combination of such metallic nanostructures can also support Fano-like resonances. ${ }^{2-4}$ These resonances arise from the interference between a nonradiative mode and a continuum of radiative waves, and are distinguished from their Lorentzian counterpart by a characteristic asymmetric line shape. They have attracted significant attention because quasi-localized modes with reduced radiative damping can be excited, allowing in particular the development of plasmonic nanolasers. ${ }^{5}$ They also have been observed as extraordinary transmission through metallic films, ${ }^{6,7}$ in metallic photonic crystals, ${ }^{8}$ and have been used to explain Wood's anomalies in metallic gratings. ${ }^{9}$ A stronger field enhancement than that of conventional plasmon modes also makes Fano-like resonances promising for applications such as refractive index sensing for the development of chemical or biological sensors. ${ }^{10-13}$ Their integration with nonlinear and phasechange media finds applications in switching and electro-optics. ${ }^{14-16}$

The control of the line shape of Fano-like resonances for specific applications remains however a challenging task and requires a theory able to understand the underlying physical mechanisms. Numerical and experimental data are usually fitted and analyzed with the two coupled mechanical oscillators ${ }^{17,18}$ or other intuitive phenomenological models. ${ }^{19-21}$ In plasmonic systems, Fano resonances arise from the interaction between a nonradiative (dark) mode and a continuum generated by a radiative (bright) mode in lossy materials, and cannot be completely described by these models.

\section{ABSTRACT}
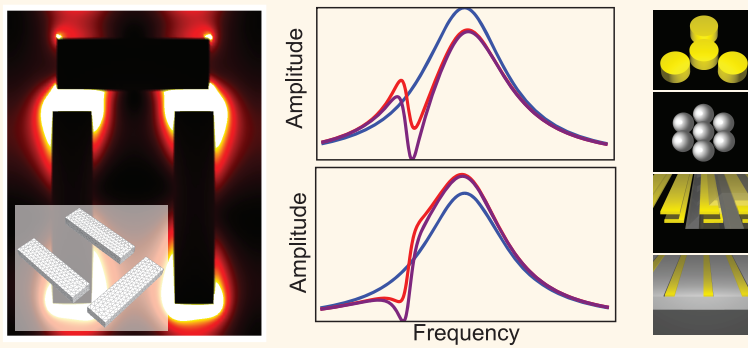

The optical properties of plasmonic nanostructures supporting Fano resonances are investigated with an electromagnetic theory. Contrary to the original work of Fano, this theory includes losses in the materials composing the system. As a result, a more general formula is obtained for the response of the system and general conclusions for the determination of the resonance parameters are drawn. These predictions are verified with surface integral numerical calculations in a broad variety of plasmonic nanostructures including dolmens, oligomers, and gratings. This work presents a robust and consistent analysis of plasmonic Fano resonances and enables the control of their line shape based on Maxwell's equations. The insights into the physical understanding of Fano resonances gained this way will be of great interest for the design of plasmonic systems with specific spectral responses for applications such as sensing and optical metamaterials.

KEYWORDS: plasmonics - optical nanocavities - Fano resonances · electromagnetically induced transparency · Wood anomalies

The quantum mechanical theory developed by Fano to explain autoionization of atoms provides a wave-like interpretation of asymmetric resonances, ${ }^{22}$ which can be adapted to a Lorentzian distribution of the continuum. ${ }^{23,24}$ In a quantum mechanical description, intrinsic losses are described by transitions to a continuum that do not interact with the discrete state (for instance the generation of phonons), whereas Maxwell's equations use the average properties of matter in the effective electric permittivity and magnetic permeability. When considering the complex electric permittivity of metals, the operator equivalent to the quantum mechanical Hamiltonian is not hermitian and the theory * Address correspondence to
olivier.martin@epfl.ch.

Received for review August 18, 2011 and accepted October 25, 2011.

Published online October 25, 2011 $10.1021 / \mathrm{nn} 203173 \mathrm{r}$

() 2011 American Chemical Society 
of Fano is not applicable. To have a consistent and general analysis and understanding of the classical phenomenon of Fano-like resonances in plasmonic nanostructures, a theory based on Maxwell's equations has to be developed. The Feshbach formalism in nuclear physics uses projection operators to model the interaction between open and closed channels ${ }^{25,26}$ and has been used as a starting point to build an electromagnetic theory of Fano-like resonances in plasmonic nanostructures. ${ }^{27}$ This theory introduces four resonance parameters which are the central frequency $\omega_{a}$, the spectral width $W_{a}$, the asymmetry parameter $q$ introduced by Fano in the quantum theory, ${ }^{22}$ and an additional parameter $b$, which quantifies the modulation damping of the resonance by intrinsic losses. ${ }^{27}$ The exact analytical expressions derived for these parameters describe the electromagnetic interactions underlying plasmonic Fano resonances.

In this work, we show that the general line shape of Fano-like resonances derived from Maxwell's equations in our previous work ${ }^{27}$ describes a broad variety of plasmonic systems. We also provide closed-form analytical formulas for the resonance parameters in terms of electromagnetic interactions, and analyze how they can be engineered in plasmonic nanostructures. In particular, the resonance spectral position and width are defined in a consistent manner that takes into account the existence of the two individual modes and their interaction. First, the formula for the resonance is introduced with its different parameters, and then discussed with several examples of plasmonic nanostructures: dolmen nanostructures, double metallic gratings, plasmonic quadrumers and heptamers, and metallic photonic crystals. For each example, a spectrum is calculated with a surface integral equations method ${ }^{28,29}$ and fitted with an analytical formula describing a background spectrum modulated by the Fano-like line shape.

\section{LINE SHAPE OF PLASMONIC FANO RESONANCES}

In this section, we expose the final results of the electromagnetic theory of plasmonic Fano resonances developed in our previous work ${ }^{27}$ and introduce the quantities that will form the basis of the following discussion. Fano-like resonances are built from the interference between a continuum of radiative waves and a nonradiative (dark) mode that spectrally and spatially overlap. Two pathways have to be considered: the direct excitation of continuum and the excitation of the dark mode through its coupling to the continuum (Figure 1a). The interference between these two pathways leads to a spectrum with a Fano-like asymmetric line shape: ${ }^{27}$

$$
\sigma_{\mathrm{a}}(\omega)=\frac{\left(\frac{\omega^{2}-\omega_{\mathrm{a}}^{2}}{2 W_{\mathrm{a}} \omega_{\mathrm{a}}}+q\right)^{2}+b}{\left(\frac{\omega^{2}-\omega_{\mathrm{a}}^{2}}{2 W_{\mathrm{a}} \omega_{\mathrm{a}}}\right)^{2}+1}
$$

where $\omega_{\mathrm{a}}$ is the resonance central spectral position, $W_{\mathrm{a}}$ gives an approximation of its spectral width in frequency units for $W_{a} \ll \omega_{a} q$ is the asymmetry parameter, and $b$ is the modulation damping parameter originating from intrinsic losses. The frequency-dependent phase difference between the direct and indirect pathways leads to both a destructive and a constructive interference at the origin of the asymmetric nature of the resonance. The dielectric permittivity of metals has an imaginary part accounting for intrinsic losses. In this case, the dark mode's resonance frequency $\omega_{d}+$ $i \gamma_{d}$ has an imaginary part, and the phase difference between the two direct and indirect pathways does not lead to a complete destructive or constructive interference (Figure 1a). This effect is quantified by the modulation damping parameter $b$. The parameters $q$ and $b$ are shape parameters describing the Fano-like interference, and as such they depend on how the resonance is monitored. If the asymmetry parameter $q$ is equal to zero, the resonance is symmetric and appears as a dip in the spectrum with minimal value $\sigma_{\mathrm{a}}\left(\omega_{\mathrm{a}}\right)=b$. The quantity $W_{\mathrm{a}}$ is defined such that $\sigma_{\mathrm{a}}\left(\omega_{\mathrm{a}} \pm W_{\mathrm{a}}\right) \simeq(1+b) / 2$ for $W_{\mathrm{a}} \ll \omega_{\mathrm{a}}$, where $(1+b) / 2$ is the average value between the modulation minimum and its asymptotic value.

The continuum of radiative waves can be constructed from the radiative (bright) mode of a plasmonic nanostructure (Figure $1 \mathrm{~b}$ ). The bright mode's resonance strength follows a symmetric pseudoLorentzian line shape as a function of the frequency $\omega$ :

$$
\sigma_{\mathrm{s}}(\omega)=\frac{a^{2}}{\left(\frac{\omega^{2}-\omega_{\mathrm{s}}^{2}}{2 W_{\mathrm{s}} \omega_{\mathrm{s}}}\right)^{2}+1}
$$

where $a$ is the maximum amplitude of the resonance, $\omega_{\mathrm{s}}$ is the resonance frequency, and $W_{s}$ gives an approximation of its spectral width in frequency units for $W_{\mathrm{s}} \ll \omega_{\mathrm{s}}$. The quantity $W_{s}$ gives an approximation of the resonance spectral width and is expressed in frequency units. The symmetric resonance (SR) given by eq 2 builds a continuum supporting a Fano-like resonance (also called superradiant envelope ${ }^{13}$ ). The structure generating the $\mathrm{SR}$ is placed in close vicinity to another one supporting the dark mode; ${ }^{12,13,30}$ alternatively, one can also use the strong coupling of two modes hybridizing into a bright and a dark mode. ${ }^{31-35}$ The resonance strength of the entire system represents the measurable quantity and is given by the product of the SR $\sigma_{\mathrm{s}}$ eq 2 with the asymmetric resonance $(\mathrm{AR}) \sigma_{\mathrm{a}}$ :

$$
\sigma_{\mathrm{t}}(\omega)=\sigma_{\mathrm{s}}(\omega) \sigma_{\mathrm{a}}(\omega)
$$

The analytical function $\sigma_{\mathrm{t}}$ can be used to fit various theoretical or experimental spectra including, for example, reflectance, transmittance, extinction, absorbance, radar backscattering, forward scattering, or total cross section. 

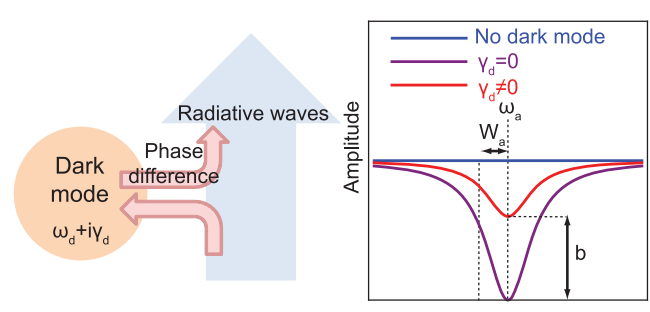

Frequency

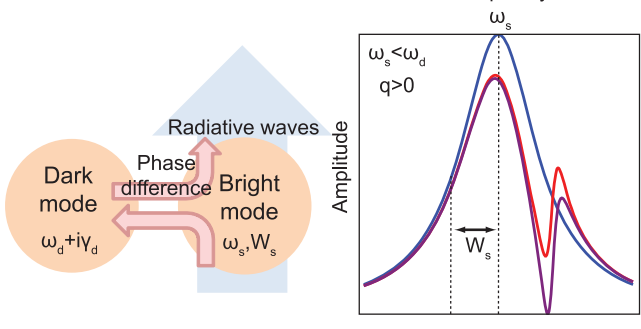

Frequency
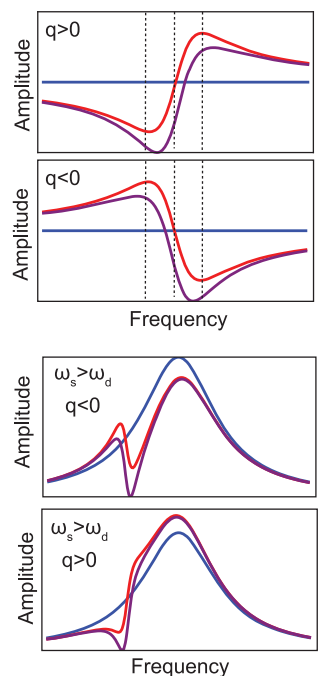

a

Figure 1. (a) Mechanism of Fano-like interferences between a resonant dark mode with complex resonance frequency $\omega_{\mathbf{d}}+$ $\mathrm{i} \gamma_{\mathrm{d}}$ and a flat continuum of radiative waves. Two pathways have to be considered: the direct excitation of continuum and the excitation of the dark mode through its coupling to the continuum. The frequency-dependent phase difference between the direct and indirect pathways leads to both a destructive and a constructive interference. The resulting line shape modulates the continuum and satisfies eq 1, where $\omega_{\mathrm{a}}$ is the Fano-like resonance frequency, $W_{\mathrm{a}}$ is its spectral width, $q$ is the asymmetry parameter, and $b$ is the modulation damping parameter. (b) Fano-like interference between a resonant dark mode and a bright mode with an amplitude satisfying eq 2 with central frequency $\omega_{\mathrm{s}}$ and spectral width $W_{\mathrm{s}}$.

This enables the retrieval of the modes' respective resonance frequency and width, as well as their spatial overlap. Analytical expressions for the resonance parameters are derived in the case of a SR line shape given by eq 2 (Methods), and they will be taken as references to explain and discuss the various effects observed in the following section. Let us mention that in most of the examples studied in this work, and generally in most experimentally relevant plasmonic systems, the SR line shape is given by eq 2 because the radiative waves are generated by a plasmon mode. It will also be shown that an arbitrary background profile can be chosen for eq 3. The approach summarized in this section represents a physically meaningful, general, and consistent way to characterize a Fano-like line shape from a spectrum.

\section{RESULTS AND DISCUSSIONS}

In this section, we verify the validity of the electromagnetic theory outlined in the previous section for a broad variety of systems that are known to support Fano-like resonances. For each structure investigated, a scattering spectrum is numerically computed using the surface integral formulation for periodic nanostructures. $^{28,29}$ The spectrum is then fitted with eq 3 following the procedure described in the Methods section, and the values of the extracted fit parameters are discussed and reported in Table 1. As an illustration of the tunability of the different AR parameters, the dolmen nanostructure appears to be a very didactic and intuitive example. It will be followed by plasmonic oligomers and a metallic double grating in which plasmon hybridization plays a role. ${ }^{36}$ We will finally study the case of metallic photonic crystals deposited
TABLE 1. Value of the Different Parameters Obtained by a Fit with Equation 3 of the Spectra in Figures 2-7, Except for Figure $6 e$ and Figure $7 b$ (See Text)

\begin{tabular}{|c|c|c|c|c|c|c|c|}
\hline & $a$ & $\omega_{s}(\mathbf{e V})$ & $W_{\mathrm{s}}(\mathrm{eV})$ & $\omega_{\mathrm{a}}(\mathrm{eV})$ & $W_{\mathrm{a}}(\mathrm{eV})$ & $q$ & $b$ \\
\hline Figure $2 b$ & 1.000 & 1.284 & 0.121 & 1.080 & 0.034 & -0.939 & 0.304 \\
\hline Figure $3 a$ & 1.000 & 1.287 & 0.120 & 1.079 & 0.038 & -0.870 & 0.397 \\
\hline Figure $3 c$ & 0.934 & 1.269 & 0.130 & 1.117 & 0.023 & -0.373 & 0.663 \\
\hline Figure $3 e$ & 1.000 & 1.248 & 0.130 & 1.554 & 0.025 & 1.297 & 0.148 \\
\hline Figure $4 b$ & 0.309 & 1.932 & 0.028 & 1.681 & 0.030 & -2.826 & 1.385 \\
\hline Figure $4 c$ & 0.289 & 1.848 & 0.044 & 1.670 & 0.027 & -1.096 & 0.257 \\
\hline Figure $4 d$ & 0.265 & 1.812 & 0.054 & 1.692 & 0.021 & -0.490 & 0.258 \\
\hline Figure 4e & 0.944 & 1.845 & 0.044 & 1.690 & 0.039 & -1.355 & 0.586 \\
\hline Figure $5 b$ & 0.149 & 2.688 & 0.115 & 2.870 & 0.058 & 1.032 & 0.135 \\
\hline Figure $6 b$ & 0.836 & 1.883 & 0.405 & 1.383 & 0.137 & 0.666 & 0.267 \\
\hline Figure $6 c$ & 0.865 & 1.823 & 0.334 & 1.422 & 0.120 & 0.594 & 0.338 \\
\hline Figure $6 \mathrm{~d}$ & 0.913 & 1.565 & 0.431 & 1.754 & 0.130 & -0.058 & 0.183 \\
\hline Figure 6e LF & 0.921 & 1.828 & 0.333 & 1.196 & 0.150 & 0.326 & 0.775 \\
\hline Figure 6e HF & & & & 1.962 & 0.135 & 0.124 & 0.363 \\
\hline Figure $7 \mathrm{~b}$ & & & & 1.986 & 0.007 & 1.493 & 1.238 \\
\hline Figure $7 c$ & 1.000 & 1.547 & 0.280 & 2.110 & 0.014 & 0.940 & 0.296 \\
\hline
\end{tabular}

on a waveguide slab. Each of the structures investigated exhibits particular advantageous features of Fano resonances such as modulation depth, width, asymmetry, isotropy, or the fabrication process used.

Dolmen Nanostructures. We first consider dolmen plasmonic nanostructures made of three metallic beams arranged as in Figure 2a. The two parallel beams (numbers 2 and 3 ) of a single dolmen support a quadrupolar dark mode, while the third perpendicular beam 

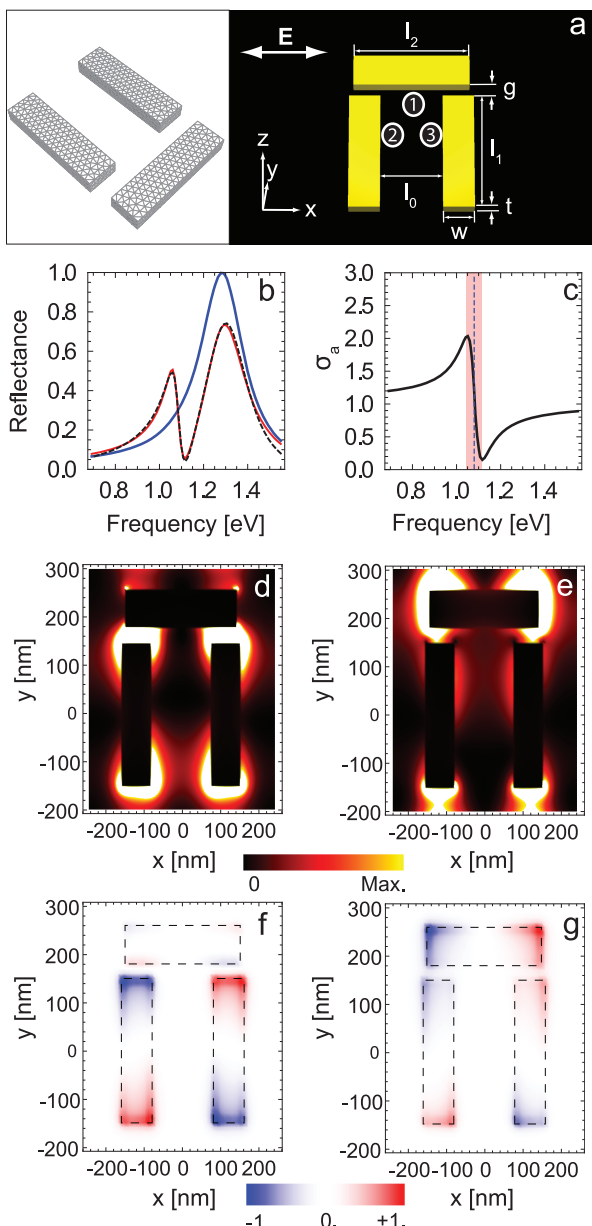

Figure 2. Reflectance of dolmen plasmonic nanostructures under normal illumination with the electric field polarized along $x$. (a) Structure dimensions and discretization: $w=$ $40 \mathrm{~nm}, I_{0}=160 \mathrm{~nm}, t=80 \mathrm{~nm}, g=30 \mathrm{~nm}$ and $I_{1}=I_{2}=300 \mathrm{~nm}$. The dolmens material is chosen to satisfy the Drude model with plasma frequency $\omega_{\mathrm{p}}=1.37 \times 10^{16} \mathrm{~s}^{-1}$ and damping $\gamma=0.009 \omega_{p}$, describing the dielectric permittivity of gold. (b) (Black dashed) numerical simulations; (red thick) fit with $\sigma_{\mathrm{t}}$ (eq 3); (blue thin) background resonance $\sigma_{\mathrm{s}}$ extracted from the fit (eq 2). (c) Respective asymmetric line shapes $\sigma_{\mathrm{a}}$ extracted from the fit (eq 1); the blue dashed line and red rectangle show respectively the resonance frequency $\omega_{a}$ and width $W_{\mathrm{a}}$. $(\mathbf{d}-\mathrm{g})$ Normalized near-field distribution of the electric field intensity in the $z$-symmetry plane at frequency (d) $1.32 \mathrm{eV}$ and (e) $1.13 \mathrm{eV}$. (f,g) Normalized near-field distribution of the $z$-component of the instantaneous electric field $5 \mathrm{~nm}$ above the array at frequency (f) $1.13 \mathrm{eV}$ and (g) $1.32 \mathrm{eV}$, revealing the quadrupolar (f) and dipolar (g) distributions of the modes. At the frequency of $1.32 \mathrm{eV}$, the dipolar and quadrupolar modes coexist.

(number 1) supports a dipolar bright mode (Figure 2f,g). The numerically computed spectrum of the unperturbed bright mode is shown in our previous work. ${ }^{27}$ The interference between the two modes leads to a Fano-like resonance. ${ }^{11,12,30,32,37}$ Figure $2 a$ shows the unit cell of the dolmens arranged in a two-dimensional array with period $500 \mathrm{~nm}$ and placed in vacuum. In Figure $2 b$, the reflectance spectrum of the array under normal illumination is calculated. Using the procedure described under Methods, we can fit this reflectance spectrum to retrieve the underlying electromagnetic structure. The SR is centered around $\omega_{\mathrm{s}}=1.284 \mathrm{eV}$ and has a spectral width of $W_{s}=0.121 \mathrm{eV}$ (Table 1). These parameters $a, \omega_{s}$, and $W_{s}$ are used to reconstruct the SR of the bright mode (blue curve in Figure 2b). The procedure adopted here is slightly different from our previous work, ${ }^{27}$ where the SR was numerically obtained from the reflectance of an array of single beams: the bright mode was assumed to be unperturbed by variations of the structure geometry. In Figure $2 c$, the $A R$ is reconstructed from the numerical values of the parameters $\omega_{\mathrm{a}}, W_{\mathrm{a}}, q$, and $b$ in Table 1. It is centered at a frequency of $1.08 \mathrm{eV}$, slightly detuned from the SR resonance frequency, and has a width of $0.034 \mathrm{eV}$. Even though the AR central frequency $\omega_{\mathrm{a}}$ is not located at a particular point of the total spectrum (neither a local minimum or a maximum), its position is consistently defined by eq 3 which considers the collective excitation of the two modes. Calculations of the electric intensity in the vicinity of the dolmen nanostructure shows that the value of the AR central frequency obtained from the fit exactly corresponds to the resonant excitation of the dark mode. ${ }^{38}$ The AR frequency is shifted from the isolated dark mode's resonance frequency $\omega_{\mathrm{d}}=1.14 \mathrm{eV}$. A quantitative comparison between the AR and SR widths can also be made: in this case, the AR width $W_{a}$ represents $28 \%$ of the SR width $W_{s}$. As expected, the AR displays a steeper amplitude modulation than its SR counterpart. However, as the AR is built upon a collective effect, its width is not only determined by the spectral width of the dark mode.

It has been shown that the field overlap between the two modes plays an important role into the control of the AR width and position. ${ }^{27}$ The modes electric field distribution in Figure 2d,e shows that the major contribution to the field overlap lies in the gap between the individual and the two parallel beams, which can be tuned by changing the gap size $g$ (Figure 2a). This is investigated in Figure $3 c, d$, where the same geometry as in Figure $2 \mathrm{~b}, \mathrm{c}$ is considered, but now with a larger gap $g=45 \mathrm{~nm}$. From Table 1, the width of the resonance is strongly influenced by such a variation of the gap size, going from $0.034 \mathrm{eV}$ for $g=30 \mathrm{~nm}$ in Figure $2 \mathrm{~b}$ to $0.023 \mathrm{eV}$ for $g=45 \mathrm{~nm}$ in Figure $3 \mathrm{c}$. This effect of the coupling to the AR line shape has also been observed in terahertz split ring resonators, where the control of the coupling of the dark mode to the bright mode is performed by symmetry breaking. ${ }^{39}$ The increase of the modes coupling decreases the time spent for the power transfer through the indirect pathway and results in a spectral broadening of the AR. The field overlap is also at the origin of a spectral shift of the AR frequency from the dark mode resonance frequency eq 4. Losses engineering is another way of optimizing the AR parameters. In Figure 3a, the Drude damping parameter used for the metal is increased 

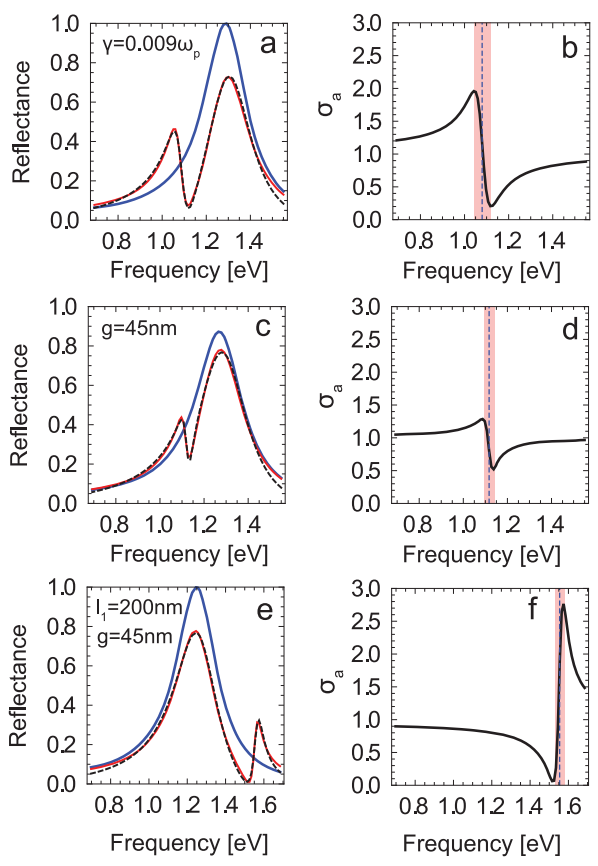

Figure 3. Influence of electromagnetic interactions on the resonance line shape of dolmen nanostructures (geometry of Figure 2): (a,b) modified Drude damping $\gamma=0.0012 \omega_{\mathrm{p}}$ for beams 2 and $3,(c, d)$ modified gap size $g=45 \mathrm{~nm},(e, f)$ modified gap size $g=45 \mathrm{~nm}$ and length $I_{1}=200 \mathrm{~nm}$. $(\mathrm{a}, \mathrm{c}, \mathrm{e})$ (Black dashed) numerical simulations; (red thick) fit with $\sigma_{\mathbf{t}}$ (eq 3); (blue thin) background resonance $\sigma_{\mathrm{s}}$ extracted from the fit (eq 2). (b,d,f) Respective asymmetric line shapes $\sigma_{\mathrm{a}}$ extracted from the fit (eq 1); the blue dashed line and red rectangle show, respectively, the resonance frequency $\omega_{\mathrm{a}}$ and width $W_{\mathrm{a}}$.

in the beams 2 and 3 . As predicted from the electromagnetic theory, intrinsic losses damp the AR and strongly affect the AR width, asymmetry, and modulation depth (compare the first and second lines in Table 1). A very small shift of the AR frequency is also observed.

The modes coupling has a strong influence on the shape parameters $q$ and $b$ (Methods). In the presence of intrinsic losses, the interference between the direct and indirect pathways is not total and the Fano modulation is damped. The $b$ parameter quantifies this effect and is given by the ratio between the intensity lost to the metallic structure and the intensity transferred from the bright mode to the dark mode. If the coupling between the two modes is too weak compared to intrinsic losses, almost no modulation is observed. The parameter $b$ increases from 0.304 for a gap size of $g=30 \mathrm{~nm}$ (Figure 2b) to 0.663 for $g=45 \mathrm{~nm}$ (Figure $2 \mathrm{~b}$ ): this gives a quantitative description of a phenomenon that has been observed in previous works on dolmen nanostructures. ${ }^{12,37}$ An increase of the parameter $b$ is also observed with an increase of the intrinsic losses (Figure 3a). The parameter $b$ determines the maximal amount of light that can be transmitted and is therefore critical for achieving the plasmonic equivalent of electromagnetically induced transparency.
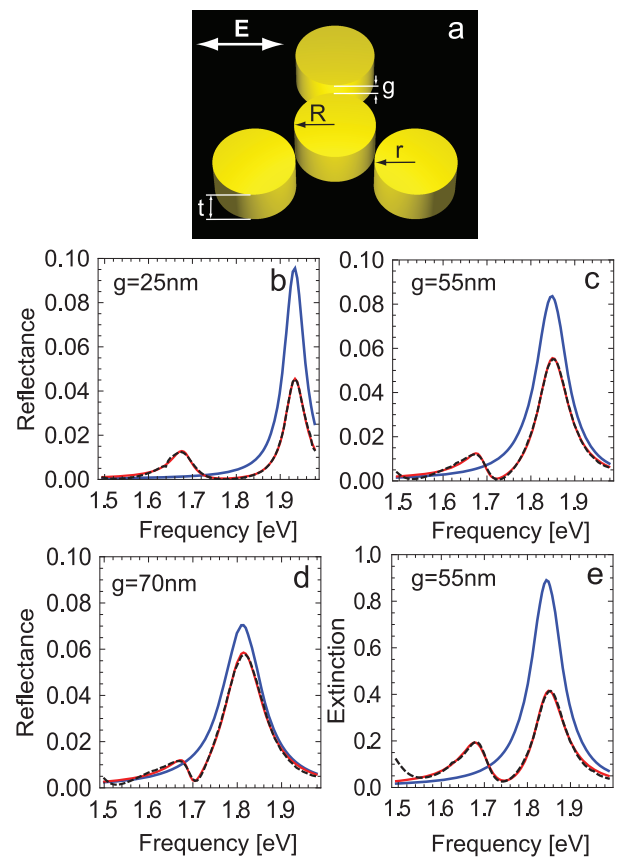

Figure 4. Optical properties of a two-dimensional array of gold quadrumers at normal incidence. (a) Geometry of a single structure with dimensions $R=80 \mathrm{~nm}, r=75 \mathrm{~nm}$, and $t=80 \mathrm{~nm}$. The quadrumers are made of metal with Drude model with plasma frequency $\omega_{\mathrm{p}}=1.37 \times 10^{16} \mathrm{~s}^{-1}$ and damping $\gamma=4.08 \times 10^{13} \mathrm{~s}^{-1}$, placed in a two-dimensional array of period $1400 \mathrm{~nm}$, and embedded in a dielectric background of refractive index 1.25 . (b-d) Reflectance and (e) extinction. (b-e) (Black dashed) numerical simulations; (red thick) fit with $\sigma_{\mathrm{t}}$ (eq 3 ); (blue thin) background resonance $\sigma_{\mathrm{s}}$ extracted from the fit (eq 2).

The absolute value of the asymmetry parameter $q$ depends on the ratio between the intensity lost to the metallic structure and the intensity transferred from the bright mode to the dark mode: from Table 1, it decreases from 0.939 to 0.373 when the gap size is increased from 30 to $45 \mathrm{~nm}$. The sign of the asymmetry parameter is determined by the frequency difference between the dark and the bright mode. In Figure 3e, the length of the beams 2 and 3 is shortened from 300 to $200 \mathrm{~nm}$, resulting in a blue-shift of the dark mode's resonance frequency. As a consequence, the AR frequency is shifted from 1.117 to $1.554 \mathrm{eV}$. The bright mode's phase is reversed, and as a consequence the modulation shape: the asymmetry parameter flips its sign from -0.373 to 1.297 . It has been shown that owing to retardation effects, the quadrupolar mode can be excited under grazing incidence illumination. ${ }^{12}$ As the excitation angle $\theta$ increases, a radiative channel, which was forbidden by the periodic symmetry of the array at normal incidence, opens and the reflectance spectrum becomes that of two radiative modes rather than an asymmetric modulation of the radiative mode's amplitude. This case does not satisfy the basic assumptions of the electromagnetic theory ${ }^{27}$ and the classical oscillators model. ${ }^{18}$ Placing the dolmens in subwavelength arrays also ensures that the quadrupolar 
mode is a true dark mode, but near-field interactions with nearest neighbors have to be taken into account.

Plasmon Hybridization and Plasmonic Oligomers. Plasmonic oligomers - such as the quadrumer shown in Figure 4ause the hybridization of a circular chain of nanoparticles with a central nanoparticle to produce a dark and a bright mode, whose interference gives rise to a Fano-like resonance. ${ }^{40,41}$ Owing to the metallic character of the particles, the dark and bright modes are not completely orthogonal. Their coupling and the subsequent generation of the Fano resonance does not require a structural symmetry breaking. In the bright mode's configuration, the central particle is in phase with the outer ring's particles, whereas in the dark mode's configuration, the central particle is out of phase, reducing the overall dipole moment of the structure. A structure supporting such modes with a minimal amount of nanoparticles is a quadrumer whose outer ring is composed of three particles and carries the symmetry $D_{3 h}$ (or $C_{3 v}$ if placed on a substrate) as shown in Figure 4. The reflectance of plasmonic quadrumers placed in an array is calculated in Figure 4 panels b, c, and d for separation distances between the central particle and the outer ring of 25,55 , and $70 \mathrm{~nm}$, respectively. The electric field distribution in the structure at the AR frequency shows that the central particle oscillates out of phase with the exterior particles, therefore reducing the overall dipole moment. The bright mode is relatively narrow, but its spectral overlap with the AR ensures the validity of eq 3 . Since the two modes interfering to produce a Fano-like resonance are supported by the same structure, the SR background cannot be explicitly computed. Nevertheless, the fit of the total spectrum enables the extraction of the SR for both reflection and extinction (blue curve in Figure $4 b-e$ ) in the same way as in Figure 2 and Figure 3. Similarly to that of the dolmen nanostructure, an increase of the gap $g$ leads to a decrease of the field overlap between the two modes and consequently of the AR width (Table 1). This suggests that the bright mode resembles the resonance of the same structure without the central particle. ${ }^{41}$ The asymmetry parameter has a relatively large absolute value compared to the other structures investigated in this paper, so that the overall optical response has the appearance of two distinct peaks (Figure $4 b-d$ ). In the extinction spectrum of Figure $4 \mathrm{e}$, similar results for the spectral positions and width of the SR and AR are obtained, but the asymmetry parameter is even higher, resulting in an even more pronounced peak. This drastic change of $q$ and $b$ when comparing the reflectance and the extinction spectra of the same structure under the same illumination condition, shows that they are interference parameters: they depend not only on the near-field interactions between the bright and dark modes, but

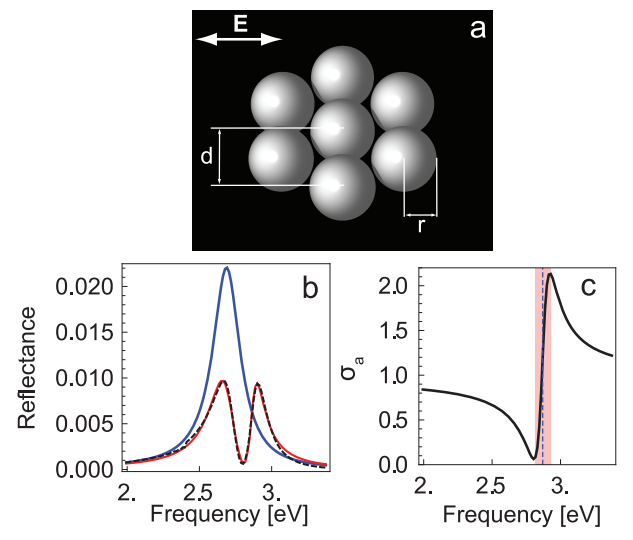

Figure 5. Reflectance of a two-dimensional array of heptamers of spherical nanoparticles at normal incidence. (a) Geometry of a single structure with dimensions $r=30 \mathrm{~nm}$ and $d=65 \mathrm{~nm}$. The heptamers are separated by a period of $900 \mathrm{~nm}$ and placed in vacuum. Experimental data for silver has been taken for the particles material. ${ }^{46}$ (b) (Black dashed) numerical simulations; (red thick) fit with $\sigma_{\mathrm{t}}$ (eq 3); (blue thin) background resonance $\sigma_{\mathrm{s}}$ extracted from the fit (eq 2). (c) Respective asymmetric line shapes $\sigma_{a}$ extracted from the fit eq 1 ; the blue dashed line and red rectangle show, respectively, the resonance frequency $\omega_{a}$ and width.

also on how the resonance is monitored (see for instance Babic et al. $^{42}$ for a discussion on the asymmetry parameter in photonic crystal slabs). This fact has been observed for the case of Mie scattering against a metallic sphere: ${ }^{2,43}$ the dipolar and quadrupolar modes of the sphere interfere together to form a Fano resonance in the vicinity of the quadrupolar mode's resonance frequency. In this case, the $q$ parameter reverses its sign depending whether a forward or backward scattering cross section is considered. For the quadrumers, a vectorial symmetry analysis shows that in-plane dipolar modes are always 2 -fold degenerate. ${ }^{44,45}$ The dark mode of the quadrumer structure is also degenerate, so that a Fano resonance at similar energies is obtained for the orthogonal polarization. A Fano-like resonance can be found in plasmonic oligomers with a higher number of surrounding particles, such as heptamers. ${ }^{35,40}$ In Figure 5, we consider an arrangement of seven silver spheres. From Table 1, the AR appears larger with this geometry than the other cases treated in this work, but it has on the other hand the lowest value of $b$. This quantitatively asserts that the line shape almost reaches zero values with a very large modulation depth. Plasmonic heptamers show a high degree of tunability by controlling the spacing, shape, and dimensions of the particles, and the various ways of breaking its high symmetry. ${ }^{35,40,41}$ How the variation of these parameters affect the shape of the AR can be quantified following the same procedure provided that no radiative channel for the dark mode is open. The heptamer structures carry the high symmetry $D_{6 h}=$ $C_{s} \otimes C_{6 v}$. Similarly to the quadrumers with $D_{3 h}$ symmetry, the bright and dark modes are 2-fold degenerate. 

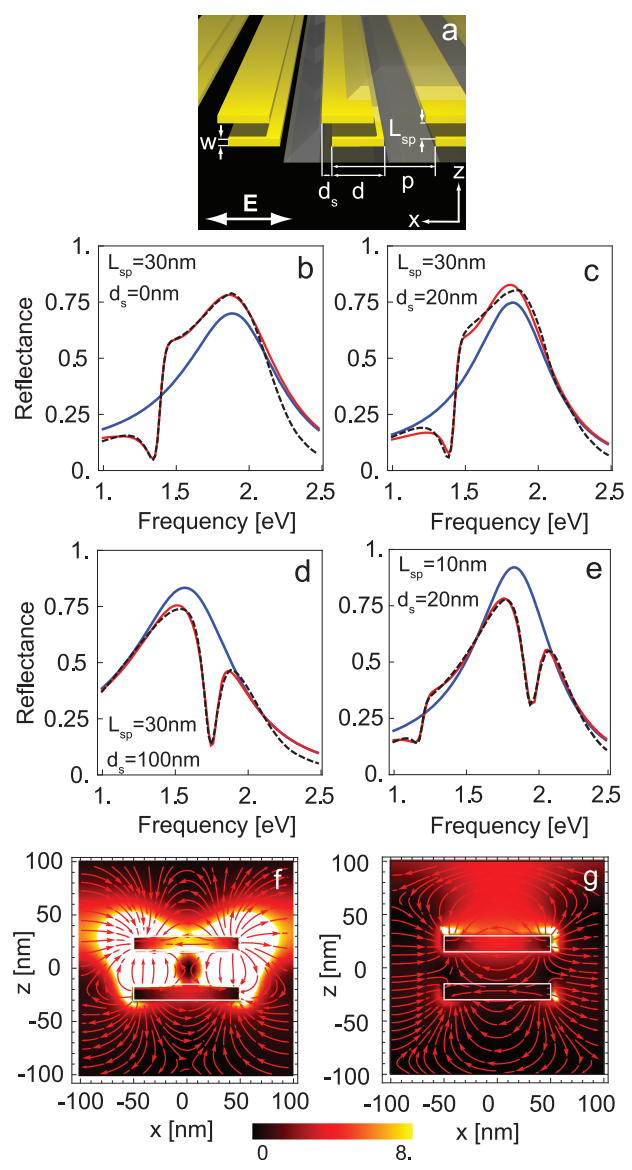

Figure 6. Reflectance of a plasmonic double grating for varying gratings separation $L_{\mathrm{sp}}$ and symmetry breaking $d_{\mathrm{s}}$ at normal incidence. (a) The structure dimensions are $d=$ $100 \mathrm{~nm}, p=200 \mathrm{~nm}$, and $w=15 \mathrm{~nm}$. The wires are made of gold (data from Johnson and Christie ${ }^{46}$ ) and embedded in a silica matrix (refractive index 1.46). (b-e) (Black dashed) numerical simulations; (red thick) fit with $\sigma_{\mathrm{t}}$ (eq 3); (blue thin) background resonance $\sigma_{\mathrm{s}}$ extracted from the fit (eq 2). In panel e, two resonances of different order occur, labeled as Low Frequency (LF) and High Frequency (HF), in which the quadrupolar and octupolar modes are respectively excited. $(\mathbf{f}-\mathbf{g})$ Near-field electric intensity distribution and instantaneous electric field lines for the geometry of panel b at (f) 1.37 and (g) $1.91 \mathrm{eV}$, respectively, revealing the distributions of the quadrupolar and dipolar modes.

A similar resonance is obtained for the orthogonal polarization. Unlike the other structures investigated in this work, plasmonic oligomers are able to generate quasi-isotropic Fano resonances. ${ }^{35-41}$

Asymmetry Reversal in Metallic Double Gratings. In the metallic double grating structure in Figure 6, the individual modes of each metallic wire hybridize to a symmetric bright mode and an antisymmetric dark mode. The offset $d_{\mathrm{s}}$ between the two wires introduces a symmetry breaking in the structure which is used to tune the resonance frequency of the two modes. ${ }^{31,47}$ The parameters used for fitting the reflectance spectra are reported in Table 1. The AR frequencies do not correspond to a particular point of the spectrum (neither a local minimum nor a local maximum) but can be

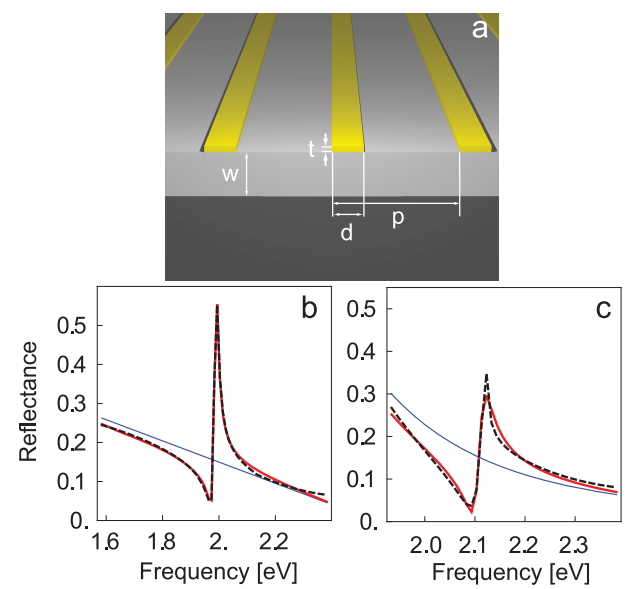

Figure 7. Reflectance of a planar metallic photonic crystal structure at normal incidence. (a) The structure dimensions are $t=20 \mathrm{~nm}, d=100 \mathrm{~nm}, p=400 \mathrm{~nm}$, and $w=140 \mathrm{~nm}$. (b) TE illumination (electric field parallel to the gold wires). (c) TM illumination (electric field perpendicular to the gold wires): (black dashed) numerical simulations; (red thick) fit with $\sigma_{\mathbf{t}}$ (eq 3); (blue thin) background resonance $\sigma_{\mathrm{s}}$ extracted from the fit (eq 2).

shown to correspond to the resonance frequency of the dark mode. ${ }^{38}$ For the bright mode, charges of identical sign are moved further apart by the symmetry breaking, resulting in a red-shift of the SR (from 1.883 to $1.565 \mathrm{eV}$ in Figure $6 \mathrm{~b}-\mathrm{d}$ ). On the other hand, for the dark mode the symmetry breaking brings charges of identical sign closer to each other, resulting in a blueshift of the AR (from 1.42 to $1.75 \mathrm{eV}$ in Figure $6 \mathrm{~b}-\mathrm{d}$. The reduction of the modes detuning makes the AR shape more symmetric. If the wires' separation Lsp is decreased to $10 \mathrm{~nm}$, the AR frequency shifts from 1.42 to $1.19 \mathrm{eV}$ because charges of opposite sign are brought close to each other; the inverse shift is observed for the bright mode. Furthermore, an additional AR appears for $d_{\mathrm{s}}=20 \mathrm{~nm}$ at $1.96 \mathrm{eV}$ (Figure 6e), resulting from the interference between an octupolar mode and the dipolar mode. ${ }^{31}$ Four additional parameters have been added to the fit in order to take this higher order AR into account. In Figure $6 b-e$, the sign of the asymmetry parameter is positive even though the $A R$ is located on the low frequency shoulder of the SR, unlike all previous examples (Figures $2-6$ ). This sign reversal is due to the antisymmetric nature of the dark modes with respect to the $z=0$ plane (Figure $6 f$ ). The phase difference between the direct and indirect pathways is opposite to the phase difference induced by a $z$-symmetric dark mode, and as a result also the sign of the asymmetry parameter (see Methods).

Excitation of First Orders in Metallic Photonic Crystals. The Fano resonance is also supported by periodic metallic crystals. ${ }^{48}$ In Figure 7, the reflectance spectra of an array of gold nanowires placed on a single-mode slab waveguide is calculated for both polarizations at normal incidence. The metallic grating on top is made of gold (data from Johnson and Christie ${ }^{46}$ ), the waveguide 
layer of ITO (refractive index 1.9), and the substrate of quartz (refractive index 1.46). In Figure $7 b$, the excitation of TE quasi-guided modes induces an AR. Another waveguide mode is excited through the grating for a TM polarization in Figure 7c. For TE polarization, the background is not created from a plasmon resonance, ${ }^{8}$ and has been fitted with the function of the frequency $\sigma_{\mathrm{s}}(\omega)=g \omega+h$, corresponding to a Taylor expansion of the background profile around the AR frequency: with such procedure, one can fit a Fano-like resonance with any background by considering its local behavior in a small frequency interval. We also stress that this spectrum cannot be fitted with Fano formula $(b=0)$. The substrate can induce a phase shift between the direct and indirect pathways that is different from an homogeneous medium and the model of a real $q$ parameter is not valid anymore. ${ }^{42}$ In such case, the modulation damping parameter also contains contributions from the imaginary part of $q$. The value of the AR parameters are reported in Table 1 together with $g=-0.27 \mathrm{eV}^{-1}$ and $h=$ 0.70 . The frequency window used for the fit is robust: the parameters remain identical up to the second digit. In TM polarization, the individual metallic wires support a plasmon resonance at lower frequency, and the coupling between plasmons and waveguide mode can form waveguide plasmon polaritons. ${ }^{48}$ In this case, the background can be fitted with eq 2 . With the dielectric character of the ITO layer chosen in the simulations, the waveguide modes do not have strong intrinsic losses and even a very weak coupling from the metallic grating is sufficient to induce the AR. As a result, the two ARs of Figure 7 panels $b$ and $c$ are the narrowest with $W_{a}=0.007$ $\mathrm{eV}$ and $W_{\mathrm{a}}=0.014 \mathrm{eV}$, respectively, but do not correspond to a localized dark mode. It is worth mentioning that the excitation of quasi-guided first order waves from a metallic grating, also known as the Wood or Rayleigh anomaly, has been recently unified with the concept of Fano interferences, ${ }^{9,49}$ and is at the origin of extraordinary transmissions in subwavelength hole arrays. ${ }^{6,7}$ All these physical phenomena fall into the range of applications of eq 1.

\section{CONCLUSIONS}

The optical properties of plasmonic nanostructures supporting Fano resonances have been investigated with surface integral numerical simulations and compared to the predictions of an electromagnetic theory. By fitting the analytical formula to the numerical simulations, we have shown that the line shape of Fano resonances satisfies the predictions of the electromagnetic theory in a broad variety of plasmonic nanostructures, and that this procedure is applicable for any continuum of radiative waves.

In most plasmonic nanostructures, Fano-like resonances arise from the interference between a radiative and a nonradiative mode. We have derived closed-form analytical expressions of the resonance parameters for this particular case and quantitatively verified their behavior. Our results indicate that the frequency position of the asymmetric resonance is determined by the dark mode's frequency and perturbed by its coupling to the bright mode. The width of the asymmetric resonance is mainly determined by the modes coupling, while intrinsic losses drastically affect the modulation depth and asymmetry of the resonance. The asymmetry and modulation damping parameters describe the interference and depend on the observable that is chosen to monitor the resonance, for instance reflectance or extinction spectra.

This work presents a general, robust, and consistent analysis of plasmonic Fano resonances and enables the control of their line shape based on Maxwell's equations. The insights into the physical understanding of Fano resonances gained this way will be of great interest for the design of plasmonic systems with specific spectral responses for applications such as sensing and optical metamaterials.

\section{METHODS}

We would like to refer the reader to the work where the electromagnetic theory describing Fano resonances in plasmonic systems is derived. ${ }^{27}$ This theory leads to equations 1 and 3. In this section, we show how to retrieve the characteristic resonance parameters from fitting eq 3 with numerical simulations. Numerical simulations were performed with a surface integral formulation method for periodic nanostructures. ${ }^{28,29}$ Open boundary conditions and illumination conditions are explicitly included in the equations. To compute reflectance spectra, the scattered electric field is sampled sufficiently far away from the array (typically 30 wavelengths). The zeroth order of its discrete Fourier transform in the unit cell is calculated and multiplied by its complex conjugate. For extinction spectra, the transmittance is first calculated from the total electric field (incident + scattered) in a similar way as the reflectance, but on the other side of the array. The extinction is then calculated as to be $1-$ transmittance. The fit of the spectra with eq 3 is performed in most cases by variations of the seven parameters $a, \omega_{\mathrm{s}}, W_{\mathrm{s}}, \omega_{\mathrm{a}}, W_{\mathrm{a}}, q$, and $b$ (eq 1 and 2). To fulfill energy conservation, the SR amplitude $a$ is constrained to be inferior to 1. In Figure 6e with two ARs, the fit has been performed with two sets of four parameters and a set of three parameters for the background. In Figure $7 b$, only two parameters have been used for fitting the background as it is not generated by a plasmon resonance. These two parameters correspond to a Taylor expansion of the background reflectance around the AR frequency.

The AR parameters in eq 1 can be calculated analytically in the far-field for the particular case where the optical response of the bright mode satisfies eq 2 . The resonance frequency of the dark mode is $\omega_{d}+i \gamma_{d}$, where $\gamma_{d}$ is related to intrinsic losses in the metallic scatterers. The AR parameters are assumed to be constant in the AR spectral region and are evaluated at $\omega_{\mathrm{d}}$. The AR frequency is related to the real part of the dark mode's resonance frequency $\omega_{d}$ by

$$
\omega_{\mathrm{a}}^{2}=\omega_{\mathrm{d}}^{2}+\omega_{\mathrm{d}} \Delta
$$


where $\Delta$ is the AR frequency shift due to coupling $c$ between the dark and bright modes:

$$
\Delta= \pm \frac{c^{2}\left(\omega_{\mathrm{d}}^{2}-\omega_{\mathrm{s}}^{2}\right) \Gamma_{\mathrm{s}}}{2 \omega_{\mathrm{d}}^{2}\left(\left(\omega_{\mathrm{d}}^{2}-\omega_{\mathrm{s}}^{2}\right)^{2}+\Gamma_{\mathrm{s}}^{2}\right)}
$$

where $\Gamma_{\mathrm{s}}=2 W_{\mathrm{s}} \omega_{\mathrm{s}}$ is the SR width in units of frequency squared. The quantity $W_{\mathrm{s}}$ represents an approximation of the SR width in frequency units for $W_{\mathrm{s}} \ll \omega_{\mathrm{s}}$. The parameter $\Delta$ (as well as the asymmetry parameter $q$ ) is written in an integral form involving a dyadic Green's function. The principal value of these singular integrals can be calculated using the residue theorem. The + (-) sign arises from a phase degree of freedom for the Green's function, and corresponds to a situation where the radiative continuum supported by the bright mode oscillates in (out of) phase with its reciprocal. ${ }^{19}$ In the classical oscillators model, ${ }^{18,27}$ the equivalent effect is obtained by assuming a purely imaginary coupling constant. The sign of $\Delta$ is also determined by the frequency difference between the two modes, and its magnitude linearly depends on the coupling strength, which is a signature of weak coupling. The AR width in units of frequency squared is given by

$$
\Gamma_{a}=\frac{c^{2} \Gamma_{\mathrm{s}}^{2}}{2 \omega_{\mathrm{d}}\left(\left(\omega_{\mathrm{d}}^{2}-\omega_{\mathrm{s}}^{2}\right)^{2}+\Gamma_{\mathrm{s}}^{2}\right)\left(1-\alpha \gamma_{\mathrm{d}} / c^{2}\right)}
$$

where

$$
\alpha=\frac{4 \omega_{d}^{2}\left(\left(\omega_{d}^{2}-\omega_{s}^{2}\right)^{2}+\Gamma_{s}^{2}\right)}{\left(\omega_{d}^{2}-\omega_{s}^{2}\right)^{2}+16 \gamma_{d}^{2} \omega_{d}^{4}\left(\left(\omega_{d}^{2}-\omega_{s}^{2}\right)^{2}+\Gamma_{s}^{2}\right)^{2} / c^{4}}
$$

is a constant. Previously called $\Gamma$ in the original theory, ${ }^{28}$ the quantity $\Gamma_{\mathrm{a}}$ is linked to the AR width in frequency units $W_{\mathrm{a}}$ by $\Gamma_{\mathrm{a}}=2 W_{\mathrm{a}} \omega_{\mathrm{a}}$. The asymmetry parameter $q$ and modulation damping parameter $b$ depend on the optical response that is considered for the resonance. Considering this response similar to the one in eq 2 , the $q$ parameter becomes

$$
q= \pm\left(1-\frac{\alpha \gamma_{d}}{c^{2}}\right) \frac{\omega_{d}^{2}-\omega_{s}^{2}}{\Gamma_{s}}
$$

Only the case of a real asymmetry parameter, corresponding to structures embedded in a homogeneous dielectric background, is considered in this work. For more general structures, the asymmetry parameter $q$ is expected to have imaginary parts and contribute to the AR modulation damping. ${ }^{42}$ Finally, the modulation damping parameter $b$ includes contributions of the intrinsic losses and the modes coupling:

$$
b=4 \frac{\gamma_{\mathrm{d}}^{2} q^{2}}{\Delta^{2}}=\left(1-\frac{\alpha \gamma_{\mathrm{d}}}{c^{2}}\right)^{2} \frac{16 \gamma_{\mathrm{d}}^{2} \omega_{\mathrm{d}}^{4}\left(\left(\omega_{\mathrm{d}}^{2}-\omega_{\mathrm{s}}^{2}\right)^{2}+\Gamma_{\mathrm{s}}^{2}\right)^{2}}{\Gamma_{\mathrm{s}}^{4} c^{4}}
$$

In the parameters $\Gamma_{\mathrm{a}}, q$, and $b$, intrinsic losses appear in the ratio $\gamma_{d}\left(\left(\omega_{d}^{2}-\omega_{s}^{2}\right)^{2}+\Gamma_{s}^{2}\right) / c^{2}$, that is, the ratio between the power lost in the metallic structure and the power transferred from the bright mode to the dark mode. This ratio also appears in the expressions for the AR width (eq 6) and asymmetry (eq 8) and accounts for its efficiency.

Acknowledgment. Fundings from CSEM and CCMX-Fanosense as well as stimulating discussion with M. Schnieper, A. Stuck, and P. Nordlander are gratefully acknowledged.

\section{REFERENCES AND NOTES}

1. Barnes, W. L.; Dereux, A.; Ebbesen, T. W. Surface Plasmon Subwavelength Optics. Nature 2003, 424, 824-830.

2. Luk'yanchuk, B.; Zheludev, N. I.; Maier, S. A.; Halas, N. J.; Nordlander, P.; Giessen, H.; Chong, C. T. The Fano Resonance in Plasmonic Nanostructures and Metamaterials. Nat. Mater. 2010, 9, 707-715.

3. Miroshnichenko, A. E.; Flach, S.; Kivshar, Y. S. Fano Resonances in Nanoscale Structures. Rev. Mod. Phys. 2010, 82, 2257-2298.
4. Halas, N. J.; Lal, S.; Chang, W.-S.; Link, S.; Nordlander, P. Plasmons in Strongly Coupled Metallic Nanostructures. Chem. Rev. 2011, 111, 3913-3961.

5. Zheludev, N. I.; Prosvirnin, S. L.; Papasimakis, N.; Fedotov, V. A. Lasing Spaser. Nat. Photon. 2008, 2, 351-354.

6. Collin, S.; Vincent, G.; Haidar, R.; Bardou, N.; Rommeluere, S.; Pelouard, J.-L. Nearly Perfect Fano Transmission Resonances through Nanoslits Drilled in a Metallic Membrane. Phys. Rev. Lett. 2010, 104, 027401.

7. Garcia-Vidal, F. J.; Martin-Moreno, L.; Ebbesen, T. W.; Kuipers, L. Light Passing through Subwavelength Apertures. Rev. Mod. Phys. 2010, 82, 729-787.

8. Christ, A.; Tikhodeev, S. G.; Gippius, N. A.; Kuhl, J.; Giessen, $\mathrm{H}$. Waveguide-Plasmon Polaritons: Strong Coupling of Photonic and Electronic Resonances in a Metallic Photonic Crystal Slab. Phys. Rev. Lett. 2003, 91, 183901.

9. Sarrazin, M.; Vigneron, J. P.; Vigoureux, J. M. Role of Wood Anomalies in Optical Properties of Thin Metallic Films with a Bidimensional Array of Subwavelength Holes. Phys. Rev. B 2003, 67, 085415 .

10. Mirin, N. A.; Bao, K.; Nordlander, P. Fano Resonances in Plasmonic Nanoparticle Aggregates. J. Phys. Chem. A 2009, 113, 4028-4034.

11. Liu, N.; Weiss, T.; Mesch, M.; Langguth, L.; Eigenthaler, U.; Hirscher, M.; Soennichsen, C.; Giessen, H. Planar Metamaterial Analogue of Electromagnetically Induced Transparency for Plasmonic Sensing. Nano Lett. 2010, 10, 11031107.

12. Verellen, N.; Sonnefraud, Y.; Sobhani, H.; Hao, F.; Moshchalkov, V. V.; Van Dorpe, P.; Nordlander, P.; Maier, S. A. Fano Resonances in Individual Coherent Plasmonic Nanocavities. Nano Lett. 2009, 9, 1663-1667.

13. Verellen, N.; Van Dorpe, P.; Huang, C.; Lodewijks, K.; Vandenbosch, G. A. E.; Lagae, L.; Moshchalkov, V. V. Plasmon Line Shaping Using Nanocrosses for High Sensitivity Localized Surface Plasmon Resonance Sensing. Nano Lett. 2011, 11, 391-397.

14. Dicken, M. J.; Aydin, K.; Pryce, I. M.; Sweatlock, L. A.; Boyd, E. M.; Walavalkar, S.; Ma, J.; Atwater, H. A. Frequency Tunable Near-Infrared Metamaterials Based on $\mathrm{VO}_{2}$ Phase Transition. Opt. Express 2009, 17, 18330-18339.

15. Samson, Z. L.; MacDonald, K. F.; De Angelis, F.; Gholipour, B.; Knight, K.; Huang, C. C.; Di Fabrizio, E.; Hewak, D. W.; Zheludev, N. I. Metamaterial Electro-optic Switch of Nanoscale Thickness. Appl. Phys. Lett. 2010, 96, 143105.

16. Nikolaenko, A. E.; De Angelis, F.; Boden, S. A.; Papasimakis, N.; Ashburn, P.; Di Fabrizio, E.; Zheludev, N. I. Carbon Nanotubes in a Photonic Metamaterial. Phys. Rev. Lett. 2010, 104, 153902.

17. Alzar, C. L. G.; Martinez, M. A. G.; Nussenzveig, P. Classical Analog of Electromagnetically Induced Transparency. Am. J. Phys. 2002, 70, 37-41.

18. Joe, Y. S.; Satanin, A. M.; Kim, C. S. Classical Analogy of Fano Resonances. Phys. Scr. 2006, 74, 259-266.

19. Fan, S.; Joannopoulos, J. D. Analysis of Guided Resonances in Photonic Crystal Slabs. Phys. Rev. B 2002, 65, 235112.

20. Fan, S. H.; Suh, W.; Joannopoulos, J. D. Temporal CoupledMode Theory for the Fano Resonance in Optical Resonators. J. Opt. Soc. Am. A-Opt. Image Sci. Vis. 2003, 20, 569-572.

21. Ruan, Z.; Fan, S. Temporal Coupled Mode Theory for Fano Resonance in Light Scattering by a Single Obstacle. J. Phys. Chem. C 2010, 114, 7324-7329.

22. Fano, U. Effects of Configuration Interaction on Intensities and Phase Shifts. Phys. Rev. 1961, 124, 1866.

23. Shapiro, M. Electromagnetically Induced Transparency with Structured Multicontinua. Phys. Rev. A 2007, 75, 013424.

24. Giannini, V.; Francescato, Y.; Amrania, H.; Phillips, C. C.; Maier, S. A. Fano Resonances in Nanoscale Plasmonic Systems: A Parameter-free Modeling Approach. Nano Lett. 2011, 11, 2835-2840.

25. Feshbach, H. A Unified Theory of Nuclear Reactions. 2. Ann. Phys. 1962, 19, 287-313.

26. Bhatia, A. K.; Temkin, A. Line-Shape Parameters for P-1 Feshbach Resonances in He and Li+. Phys. Rev. A 1984, 29, 1895-1900. 
27. Gallinet, B.; Martin, O. J. F. Ab Initio Theory of Fano Resonances in Plasmonic Nanostructures and Metamaterials. Phys. Rev. B 2011, 83, 235427.

28. Gallinet, B.; Martin, O. J. F. Scattering on Plasmonic Nanostructures Arrays Modeled with a Surface Integral Formulation. Photon. Nanostruct. 2010, 8, 278-284.

29. Gallinet, B.; Kern, A. M.; Martin, O. J. F. Accurate and Versatile Modeling of Electromagnetic Scattering on Periodic Nanostructures with a Surface Integral Approach. J. Opt. Soc. Am. A 2010, 27, 2261-2271.

30. Liu, N.; Langguth, L.; Weiss, T.; Kaestel, J.; Fleischhauer, M.; Pfau, T.; Giessen, H. Plasmonic Analogue of Electromagnetically Induced Transparency at the Drude Damping Limit. Nat. Mater. 2009, 8, 758-762.

31. Christ, A.; Martin, O. J. F.; Ekinci, Y.; Gippius, N. A.; Tikhodeev, S. G. Symmetry Breaking in a Plasmonic Metamaterial at Optical Wavelength. Nano Lett. 2008, 8, 2171-2175.

32. Pryce, I. M.; Aydin, K.; Kelaita, Y. A.; Briggs, R. M.; Atwater, H. A. Highly Strained Compliant Optical Metamaterials with Large Frequency Tunability. Nano Lett. 2010, 10, 4222-4227.

33. Hentschel, M.; Saliba, M.; Vogelgesang, R.; Giessen, H.; Alivisatos, A. P.; Liu, N. Transition from Isolated to Collective Modes in Plasmonic Oligomers. Nano Lett. 2010, 10, 2721-2726.

34. Sonnefraud, Y.; Verellen, N.; Sobhani, H.; Vandenbosch, G. A. E.; Moshchalkov, V. V.; Van Dorpe, P.; Nordlander, P.; Maier, S. A. Experimental Realization of Subradiant, Superradiant, and Fano Resonances in Ring/Disk Plasmonic Nanocavities. ACS Nano 2010, 4, 1664-1670.

35. Fan, J. A.; Wu, C.; Bao, K.; Bao, J.; Bardhan, R.; Halas, N. J.; Manoharan, V. N.; Nordlander, P.; Shvets, G.; Capasso, F. Self-Assembled Plasmonic Nanoparticle Clusters. Science 2010, 328, 1135-1138.

36. Prodan, E.; Radloff, C.; Halas, N.; Nordlander, P. A Hybridization Model for the Plasmon Response of Complex Nanostructures. Science 2003, 302, 419-422.

37. Zhang, S.; Genov, D. A.; Wang, Y.; Liu, M.; Zhang, X. Plasmon-Induced Transparency in Metamaterials. Phys. Rev. Lett. 2008, 101, 047401.

38. Gallinet, B.; Martin, O. J. F. The Relation between Near-Field and Far-Field Properties of Plasmonic Fano Resonances. Opt. Express 2011, 19, 22167-22175.

39. Singh, R.; Al-Naib, I. A. I.; Koch, M.; Zhang, W. Sharp Fano Resonances in THz Metamaterials. Opt. Express 2011, 19, 6320-6327.

40. Bao, K.; Mirin, N. A.; Nordlander, P. Fano Resonances in Planar Silver Nanosphere Clusters. Appl. Phys. A-Mater. Sci. Process. 2010, 100, 333-339.

41. Hentschel, M.; Dregely, D.; Vogelgesang, R.; Giessen, H.; Liu, N. Plasmonic Oligomers: The Role of Individual Particles in Collective Behavior. ACS Nano 2011, 5 , 2042-2050.

42. Babic, L.; de Dood, M. J. A. Interpretation of Fano Lineshape Reversal in the Reflectivity Spectra of Photonic Crystal Slabs. Opt. Express 2010, 18, 26569-26582.

43. Tribelsky, M. I.; Flach, S.; Miroshnichenko, A. E.; Gorbach, A. V.; Kivshar, Y. S. Light Scattering by a Finite Obstacle and Fano Resonances. Phys. Rev. Lett. 2008, 100, 043903.

44. Gallinet, B.; Kupec, J.; Witzigmann, B.; Dupertuis, M. A. Analysis of Photonic Crystal Defect Modes by Maximal Symmetrization and Reduction. J. Opt. Soc. Am. B-Opt. Phys. 2010, 27, 1364-1380.

45. Zhang, W.; Gallinet, B.; Martin, O. J. F. Symmetry and Selection Rules for Localized Surface Plasmon Resonances in Nanostructures. Phys. Rev. B 2010, 81, 233407.

46. Johnson, P. B.; Christy, R. W. Optical-Constants of NobleMetals. Phys. Rev. B 1972, 6, 4370.

47. Christ, A.; Ekinci, Y.; Solak, H. H.; Gippius, N. A.; Tikhodeev, S. G.; Martin, O. J. F. Controlling the Fano Interference in a Plasmonic Lattice. Phys. Rev. B 2007, 76, 201405.

48. Christ, A.; Zentgraf, T.; Kuhl, J.; Tikhodeev, S.; Gippius, N.; Giessen, H. Optical Properties of Planar Metallic Photonic Crystal Structures: Experiment and Theory. Phys. Rev. B 2004, 70, 125113.
49. Genet, C.; van Exter, M. P.; Woerdman, J. P. Fano-Type Interpretation of Red Shifts and Red Tails in Hole Array Transmission Spectra. Opt. Commun. 2003, 225, 331. 